\title{
Non-invasive methods for estimating mPAP in COPD using cardiovascular magnetic resonance imaging
}

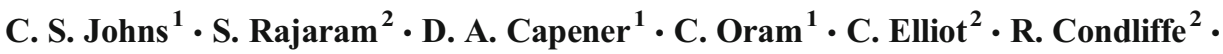 \\ D. G. Kiely ${ }^{2}$ J. M. Wild ${ }^{1}$ A. J. Swift ${ }^{1,3}$
}

Received: 1 June 2017 /Revised: 12 September 2017 / Accepted: 18 October 2017 /Published online: 16 November 2017

(C) The Author(s) 2017. This article is an open access publication

\begin{abstract}
Purpose Pulmonary hypertension $(\mathrm{PH})$ is associated with a poor outcome in chronic obstructive pulmonary disease (COPD) and is diagnosed invasively. We aimed to assess the diagnostic accuracy and prognostic value of non-invasive cardiovascular magnetic resonance (CMR) models.

Methods Patients with COPD and suspected PH, who underwent CMR and right heart catheter (RHC) were identified. Three candidate models were assessed: 1, CMR-RV model, based on right ventricular (RV) mass and interventricular septal angle; 2, CMR PA/RV includes RV mass, septal angle and pulmonary artery (PA) measurements; 3 , the Alpha index, based on RV ejection fraction and PA size.

Results Of 102 COPD patients, 87 had PH. The CMR-PA/RV model had the strongest diagnostic accuracy (sensitivity $92 \%$, specificity $80 \%$, positive predictive value $96 \%$ and negative predictive value $63 \%$, AUC $0.93, p<0.0001)$. Splitting RHCmPAP, CMR-RV and CMR-PA/RV models by $35 \mathrm{mmHg}$ gave a significant difference in survival, with log-rank chi-squared 5.03, 5.47 and 7.10. RV mass and PA relative area change were the independent predictors of mortality at multivariate Cox regression ( $p=0.002$ and 0.030 ).

Conclusion CMR provides diagnostic and prognostic information in PH-COPD. The CMR-PA/RV model is useful for
\end{abstract}

C. S. Johns

c.johns@sheffield.ac.uk

1 Academic Unit of Radiology, C floor, Royal Hallamshire Hospital, Glossop Rd, Sheffield S10 2JF, UK

2 Sheffield Pulmonary Vascular Disease Institute, Sheffield, UK

3 Insigneo, Institute of In-Silico Medicine, Sheffield, UK diagnosis, the RV mass index and PA relative area change are useful to assess prognosis.

Key Points

- Pulmonary hypertension is a marker of poor outcome in COPD.

- MRI can predict invasively measured mean pulmonary artery pressure.

- Cardiac MRI allows for estimation of survival in COPD.

- Cardiac MRI may be useful for follow up or future trials.

- MRI is potentially useful to assess pulmonary hypertension in patients with COPD.

Keywords Pulmonary Hypertension · Cardiac · Magnetic Resonance Imaging · Chronic Obstructive Pulmonary Disease $\cdot$ Cor Pulmonale

\section{Background}

Pulmonary hypertension is a predictor of death and hospitalisation [1-5] in patients with chronic obstructive pulmonary disease (COPD). Patients with pulmonary hypertension in COPD, as defined by a mean pulmonary artery pressure (mPAP) of $\geq 25 \mathrm{mmHg}$ have a 5 -year survival rate of $36 \%$ [6]. A number of studies in patients with severe COPD have shown that mild pulmonary hypertension is common: a recent study in patients with severe COPD referred for lung volume reduction surgery demonstrating pulmonary hypertension at right heart catheter in 50\% [7, 8]. More recently, with the advent of therapies for other forms of pulmonary hypertension there has been increasing interest in the subset of lung disease patients with severe (previously called "out of proportion") pulmonary hypertension, defined as an $\mathrm{mPAP} \geq 35 \mathrm{mmHg}$ or $\mathrm{mPAP} \geq 35 \mathrm{mmHg}$ with cardiac index $\leq 2.0$ [9], where a cardiovascular limitation to exercise, rather than respiratory 
limitation exists [10]. This raises the possibility that pulmonary vascular therapies may improve symptoms and outcome in this patient group [11].

The gold standard for diagnosis of pulmonary hypertension is right heart catheter (RHC), however, this is an invasive test [12]. As such, patients are screened for pulmonary hypertension with echocardiography, but unfortunately, this is less accurate in COPD where pulmonary artery pressure, when measured, is often overestimated [13, 14]. A non-invasive method for estimating mPAP in COPD patients would, therefore, be useful to help diagnose $\mathrm{PH}$, in prognostication and for possible assessment of treatment response or follow-up in clinical trials.

Several predictive cardiac magnetic resonance (CMR) imaging models have been proposed for estimation of pulmonary artery pressure [15-17]. The predictive value of these MR derived imaging models in a population of patients with suspected pulmonary hypertension in COPD remains unknown. The aim of this study was to assess the diagnostic accuracy and the prognostic value of these published models of non-invasive mPAP prediction using cardiovascular MRI.

\section{Materials and Methods}

All consecutive patients who underwent MRI at a pulmonary hypertension referral centre [18] from April 2012 to October 2015 with suspected pulmonary hypertension were assessed for inclusion. Inclusion criteria were a formal diagnosis of COPD (according to standard criteria), as per the ASPIRE (Assessing the Spectrum of Pulmonary hypertension Identified at a REferral centre) registry [18]. Patients were assessed for either obstructive spirometry (defined as FEV1/ FVC ratio of $\leq 0.70$ ) or qualitative CT evidence of emphysema, as per standard radiological practice [19]. Any patients without RHC and MRI within 90 days were excluded. Ethical approval was granted from a local ethics committee for this retrospective study, written consent was waived (ref c06/ Q2308/8).

\section{Image Acquisition}

Cardiac MRI was performed in a pulmonary hypertension tertiary referral centre [18], on a GE HDx 1.5-T whole body scanner (GE Healthcare, Milwaukee, WI, USA), using an 8channel cardiac coil, with the patient supine. Four-chamber (4Ch) and short axis (SA) cine images were acquired using a retrospectively cardiac gated multi-slice steady-state free precession (SSFP) sequence. A stack of axial images in the SA plane with slice thickness of $8 \mathrm{~mm}$ with a $2 \mathrm{~mm}$ inter-slice gap or $10 \mathrm{~mm}$ with no inter-slice gap were acquired, covering both ventricles from base to apex. The SSFP sequence parameters were: TR $2.8 \mathrm{~ms}$, TE $1.0 \mathrm{~ms}$, flip angle $50^{\circ}$, field of view 48x43.2, 256x256 matrix, $125 \mathrm{kHz}$ bandwidth, and slice thickness 8 to $10 \mathrm{~mm}$. This cardiac MRI scan protocol takes approximately $40 \mathrm{~min}$ to perform.

\section{Image Analysis and Metrics}

MR images were manually analysed by DC (a cardiac MRI radiographer of 9 years cardiac $\mathrm{MRI}$ experience) on a GE Advantage Workstation 4.4 and GE Advantage Workstation ReportCard software, with the observer blinded to all clinical information and other investigations. Left and right ventricular end-diastolic volume, end-systolic volume, right and left ventricular stroke volume and mass were calculated (all indexed to body surface area), right and left ventricular ejection fraction, ventricular mass index (RV mass divided by LV mass) [20] and interventricular septal angle were measured as previously described [15]. Right and left ventricular ejection fractions were calculated as (end diastolic volume minus end-systolic volume) divided by end diastolic volume. Maximal and minimal PA areas were manually traced, and relative area change was defined by the following equation: $\mathrm{RAC}=$ (maximum areaminimum area/minimum area) [21]. Reproducibility metrics for these cardiac MRI metrics for DC and AJS have been previously published [22]. Please see Fig. 1 for a diagram of the key imaging metrics that were measured.

\section{CMR image based models}

Previously published parametric models, developed for diagnostic and prognostic assessment in $\mathrm{PH}$, were derived from cardiac MRI metrics:

1. The CMR-RV model is based on ventricular mass index and interventricular septal angle: $\mathrm{mPAP}=-4.6+$ (interventricular septal angle x 0.23$)+($ ventricular mass index $x$ 16.3), see Fig. 1 (A and B). This model was developed in a cohort of 64 treatment naïve patients with suspected pulmonary hypertension in a tertiary referral centre. In a derivation cohort of 64 patients with suspected pulmonary hypertension, this showed good correlation with right heart catheter measured mPAP and strong diagnostic accuracy. The published threshold of $\geq 32 \mathrm{mmHg}$ had $87 \%$ sensitivity and $90 \%$ specificity for the presence of all causes of pulmonary hypertension [15].

2. The PA/RV model is similar to the CMR-RV model (above), with the addition of basic function metrics of the pulmonary artery: CMR-PA/RV $=-21.806+$ (interventricular septal angle x 0.31) + (ventricular mass index $\mathrm{x} 11.5)+($ diastolic pulmonary artery area $\mathrm{x} 0.01)-(\mathrm{PA}$ relative area change $x$ 0.22), see Fig. 1 (A, B, C and D). This model was derived in 247 patients with suspected pulmonary hypertension patients and was predictive of pulmonary hypertension in a separate prospective cohort of 
Fig. 1 Diagram showing the methodology of calculation of the cardiac MRI metrics. Pulmonary artery relative area change (a) taken from cine images taken at the level of the pulmonary trunk, perpendicular to the main pulmonary artery; septal angle (b) taken as the angle made between the insertion points of the RV to the mid septum on the endsystolic image from the short axis stack; and (c) ventricular mass index taken by segmentation of the muscle mass of the left and right ventricle on the stack of images taken in the short axis plane
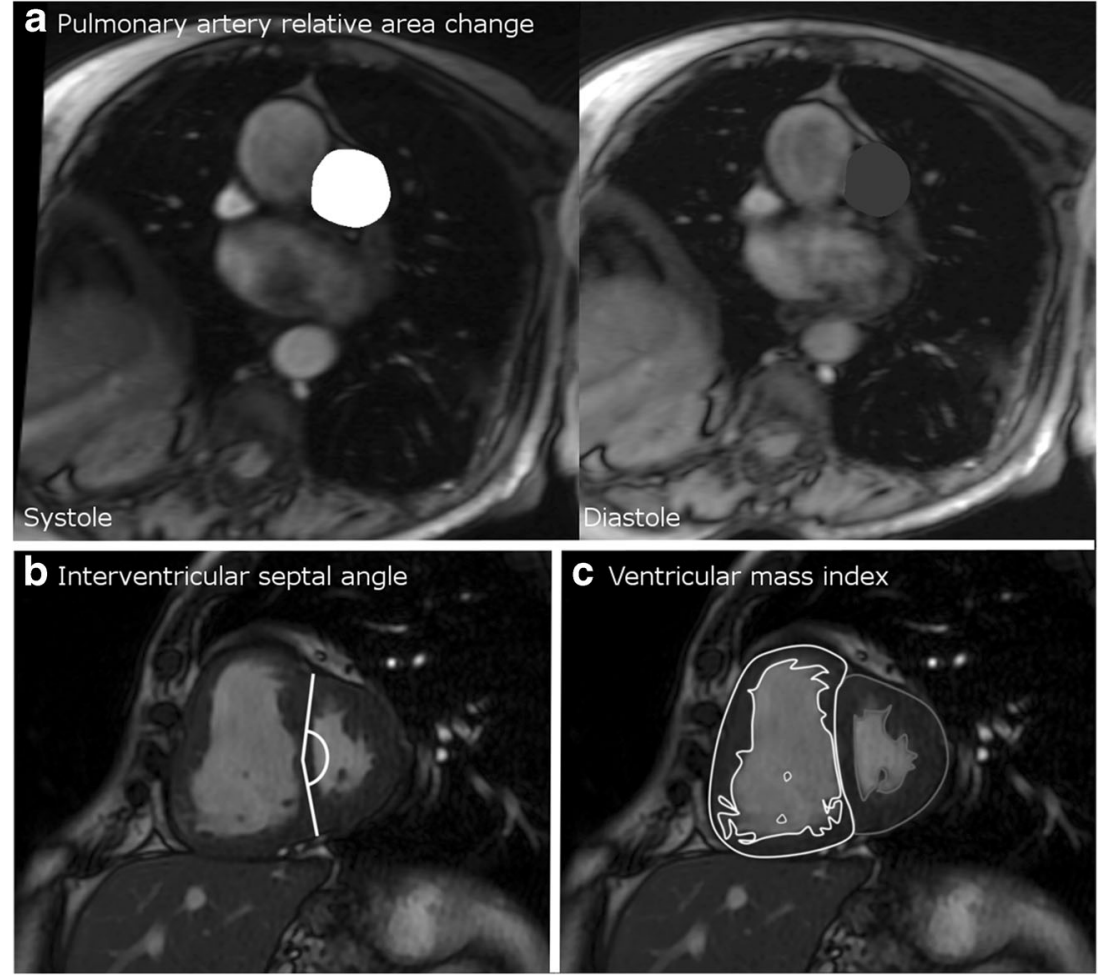

115 patients, with an area under the receiver operator curve of 0.92 [16].

3. The alpha index comprises both functional and structural information, utilising right ventricular function along with pulmonary artery size: Alpha index $=$ minimum PA area/ $\mathrm{RV}$ ejection fraction. This model was assessed in a cohort of 185 patients, with an area under ROC curve of 0.95 [17].

The diagnostic cut-off for pulmonary hypertension for CMR-RV was $\geq 32$ and alpha index $\geq 7.2$, as published in the literature [15-17]. CMR-PA/RV does not have a published threshold, so we used a threshold of $\geq 25$, as this mirrors the threshold of invasively measured mPAP at right heart catheterisation.

\section{Right Heart Catheterisation}

Right heart catheterisation was performed using a balloon tipped 7.5 Fr thermodilution catheter (Becton-Dickinson, Franklin Lakes, NJ, USA). Pulmonary hypertension was defined as a resting mPAP $\geq 25 \mathrm{mmHg}$ and severe pulmonary hypertension as a resting $\mathrm{mPAP} \geq 35 \mathrm{mmHg}$. Cardiac output was calculated using thermodilution.

\section{Statistics}

Pearson's correlation between CMR models and RHC-mPAP were calculated. The relative accuracy of the models was assessed using Bland-Altman plots. Diagnostic accuracy was calculated from the $2 \times 2$ contingency table to calculate sensitivity, specificity, negative predictive value and positive predictive value. Receiver operating characteristic (ROC) curves were constructed and the area under the ROC curve (AUC) recorded.

The interval from CMR until all cause death or census was regarded as the follow-up period. The census was performed on 25/02/2016. Log-log plots were visually inspected to ensure linearity with outcome data. Survival analysis was performed using univariate and multivariate Cox proportional hazards regression. In order to allow for comparison between each metric, this was

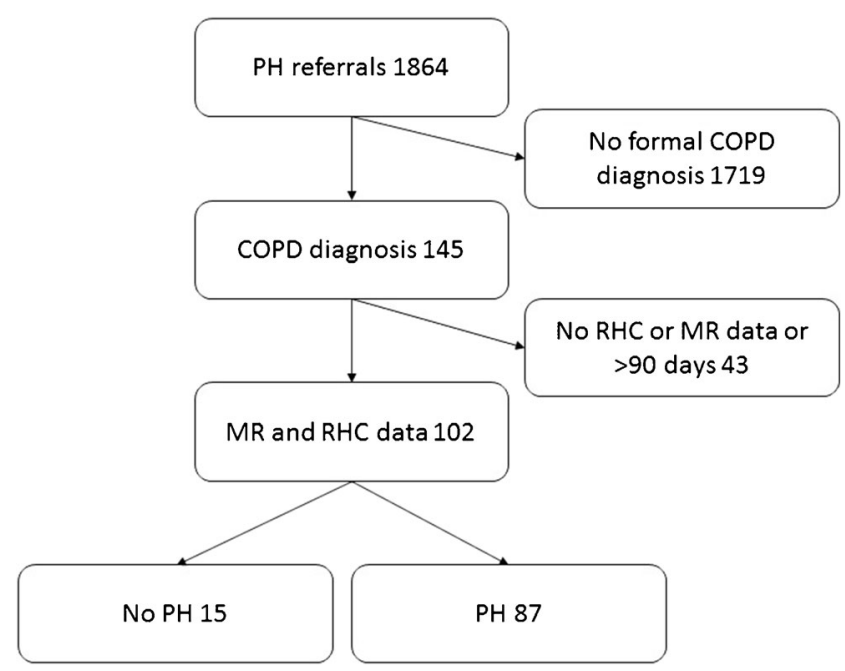

Fig. 2 Patient selection 
performed with each variable standardised as the $\mathrm{z}$ score for the population studied. Multivariate analysis was performed in a forward direction, for all variables with a statistically significant association with mortality on univariate analysis.

Kaplan-Meier plots were generated for each model and RHC measured mPAP, dichotomised by a value of 35 , and Chi square values were calculated using the Log rank test, as this is the threshold value for severe pulmonary hypertension [9]. Statistical analysis was made using SPSS 22 (IBM,
Chicago, IL, USA) and graphs were created using GraphPad Prism 7 (GraphPad Software, San Diego, CA, USA). A $p$ value of $<0.05$ was considered statistically significant.

\section{Results}

A total of 1864 patients were referred to the pulmonary hypertension centre during the period studied and underwent
Table 1 Patient demographics, mean with standard deviation with ANOVA p-value between no $\mathrm{PH}$, mild $\mathrm{PH}$ and severe $\mathrm{PH}$

\begin{tabular}{|c|c|c|c|c|c|}
\hline & All patients & No PH & $\mathrm{PH}$ & Severe PH & $\begin{array}{l}p \text {-value } \\
\text { (ANOVA) }\end{array}$ \\
\hline Number & 102 & 15 & 18 & 69 & \\
\hline \multicolumn{6}{|l|}{ Clinical Demographics } \\
\hline Age (years) & $67(12)$ & $59(17)$ & $67(12)$ & $69(9)$ & 0.012 \\
\hline \multicolumn{6}{|l|}{$\operatorname{Sex}(\mathrm{F} / \mathrm{M})$} \\
\hline \multicolumn{6}{|c|}{ WHO functional class (II,III,IV) } \\
\hline ISWT (m) & $174(169)$ & $432(264)$ & $141(101)$ & $121(87)$ & $<0.001$ \\
\hline \multicolumn{6}{|l|}{ Baseline catheter data } \\
\hline mPAP (mmHg) & $40(12)$ & $21(3)$ & $31(3)$ & $47(8)$ & $<0.001$ \\
\hline mRAP (mmHg) & $11(6)$ & $6(2)$ & $8(3)$ & $11(7)$ & 0.056 \\
\hline PAWP (mmHg) & $12(4)$ & $10(3)$ & $12(3)$ & $13(4)$ & 0.013 \\
\hline CI (Litre/m²) & $2.8(0.9)$ & $3.1(0.7)$ & $3.2(0.9)$ & $2.6(0.9)$ & 0.005 \\
\hline PVRI (Dyne.s) & $3.7(0.9)$ & $3.1(0.7)$ & $3.2(0.9)$ & $2.6(0.9)$ & $<0.001$ \\
\hline $\mathrm{SvO} 2(\%)$ & $65(9)$ & $72(8)$ & $69(5)$ & $62(9)$ & $<0.001$ \\
\hline Days RHC to MR & $5(15)$ & $5(19)$ & $5(13)$ & $5(14)$ & 0.989 \\
\hline Spirometry & $\mathrm{n}=89$ & $\mathrm{n}=9$ & $\mathrm{n}=17$ & $\mathrm{n}=63$ & \\
\hline FEV1 (\% Pred) & $66(24)$ & $76(24)$ & $58(15)$ & $66(25)$ & 0.165 \\
\hline FVC (\% Pred) & $85(22)$ & $92(20)$ & $77(15)$ & $87(24)$ & 0.155 \\
\hline FEV1/FVC & $0.55(0.15)$ & $0.63(0.20)$ & $0.51(0.10)$ & $0.55(0.15)$ & 0.179 \\
\hline TLCO (\% Pred $)$ & $30(22)$ & $80(31)$ & $33(15)$ & $21(9)$ & $<0.001$ \\
\hline \multicolumn{6}{|l|}{ Cardiac MRI } \\
\hline RV EDV index & $89(35)$ & $72(30)$ & $70(32)$ & $97(34)$ & 0.002 \\
\hline RV ESV index & $56(31)$ & $36(18)$ & $37(26)$ & $66(30)$ & $<0.001$ \\
\hline RVEF & $40(14)$ & $51(8)$ & $51(11)$ & $35(12)$ & $<0.001$ \\
\hline \multicolumn{6}{|l|}{ RV mass index } \\
\hline LV EDV index & $55(18)$ & $62(10)$ & $63(18)$ & $52(19)$ & 0.026 \\
\hline LV ESV index & $20(18)$ & $19(6)$ & $20(10)$ & $20(12)$ & 0.921 \\
\hline LVEF & $64(12)$ & $69(10)$ & $69(9)$ & $61(12)$ & 0.014 \\
\hline PA RAC & $10(8)$ & $16(14)$ & $12(8)$ & $8(6)$ & 0.008 \\
\hline VMI & $0.43(0.28)$ & $0.28(0.11)$ & $0.28(0.17)$ & $0.51(0.31)$ & 0.001 \\
\hline IVS angle & $165(24)$ & $134(10)$ & $152(14)$ & $174(20)$ & $<0.001$ \\
\hline Black blood score & $3(1)$ & $1(1)$ & $2(1)$ & $3(1)$ & $<0.001$ \\
\hline Alpha-index & $25(14)$ & $13(6)$ & $16(6)$ & $30(14)$ & $<0.001$ \\
\hline CMR-RV & $41(9)$ & $33(4)$ & $35(5)$ & $44(9)$ & $<0.001$ \\
\hline CMR-PA/RV & $35(9)$ & $23(5)$ & $30(5)$ & $39(7)$ & $<0.001$ \\
\hline
\end{tabular}

PH: pulmonary hypertension, WHO: World Health Organisation, ISWT: Incremental shuttle walk test, FEV1: Forced expiratory volume in $1 \mathrm{~s}$, RVC-mPAP: right heart catheter measured mean pulmonary artery pressure, mRAP: mean right atrial pressure, PAWP: pulmonary arterial wedge pressure, CI: cardiac index, PVRI: pulmonary vascular resistance index, $\mathrm{SvO}_{2}$ : mixed venous oxygen saturation, FVC: forced vital capacity, TLCO: transfer factor for carbon monoxide, RHC: right heart catheter, MRI: magnetic resonance imaging 
right heart catheterisation: of these 145 had a documented diagnosis of COPD from a respiratory specialist. One hundred and two had MRI and RHC within 90 days so were included in the study. There were 87 patients with pulmonary hypertension (69 of these had severe pulmonary hypertension) and 15 patients without pulmonary hypertension (please see Fig. 1 for the patient diagnostic pathway). Figure 2 shows the flow of patients within the diagnostic pathway. There were 24 with GOLD severity 1, 40 with GOLD severity 2, 18 with GOLD severity 3 and seven with GOLD severity $4 ; 13$ patients did not have spirometry results available for analysis. Of the 102 cases of COPD included in the study, 75 had spirometric evidence of airflow limitation (FEV1/FVC ratio $<0.7)$, and the remaining 17 had evidence of emphysema on CT. Table 1 shows the patient demographics and clinical characteristics for the whole group and split into no $\mathrm{PH}(\mathrm{mPAP}<25$ $\mathrm{mmHg}$ ), PH (mPAP 25 to $\leq 35 \mathrm{mmHg}$ ) and severe $\mathrm{PH}$ (mPAP $\geq 35 \mathrm{mmHg}$ ).

\section{Diagnostic accuracy}

Table 2 shows the univariate correlations of CMR imaging metrics: RV end diastolic volume index, RV end systolic volume index, RV ejection fraction, RV mass, PA diastolic area

Table 2 CMR imaging metrics and MRI model correlations with mPAP

\begin{tabular}{lcc}
\hline & \multicolumn{2}{l}{ Correlation with mPAP } \\
\cline { 2 - 3 } & $\mathrm{R}$ & $p$-value \\
\hline RVEDV index & 0.377 & $<0.0001$ \\
RVESV index & 0.482 & $<0.0001$ \\
RVEF & -0.585 & $<0.0001$ \\
RV mass & 0.372 & $<0.0001$ \\
LVEDV index & -0.304 & 0.002 \\
LVESV index & -0.029 & 0.772 \\
LVEF & -0.221 & 0.026 \\
PA diastolic area & 0.401 & $<0.0001$ \\
PA RAC & -0.344 & $<0.0001$ \\
VMI & 0.470 & $<0.0001$ \\
IVS angle & 0.710 & $<0.0001$ \\
Average PA velocity & -0.428 & $<0.0001$ \\
Black blood score & 0.603 & $<0.0001$ \\
CMR-RV & 0.689 & $<0.0001$ \\
CMR-PA/RV & 0.732 & $<0.0001$ \\
Alpha index & 0.527 & $<0.0001$ \\
\hline RVEDV & &
\end{tabular}

RVEDV: right ventricular end-diastolic volume, RVESV: right ventricular end-systolic volume, RVEF: right ventricular ejection fraction, RV: right ventricle, LVEDV: left ventricular end-diastolic volume, LVESV: left ventricular end-systolic volume, LVEF: left ventricular ejection fraction, PA: pulmonary artery, PA RAC: pulmonary artery relative area change, VMI: ventricular mass index, IVS: interventricular septum and relative area change, ventricular mass index, interventricular septal angle septal angle, average velocity and black blood score all showed statistically significant correlations with RHC mPAP. All of the models showed strong correlations with RHC measured mPAP, with $p$-values $<0.0001$. The CMR-PA/RV model showed stronger correlation with mPAP than the individual quantitative measurements alone. A scatter plot of the MRI models against RHC-mPAP is given in Fig. 3. The models all showed a stronger correlation with MPAP in the patients with mPAP $<35 \mathrm{mmHg}$ ( $\mathrm{r}$ - and $p$-values for PARV were 0.550 and 0.001, CMR-PA/RV 0.653 and $<0.001$ and alpha 0.341 and 0.052 ) than in the patients with mPAP $\geq 35 \mathrm{mmHg}$ ( $\mathrm{r}$ - and $p$-values for PA-RV were 0.431 and $<0.001$, CMR-PA/RV 0.407 and 0.001 and alpha 0.210 and 0.083 ).

Bland-Altman plots were also created to assess the accuracy of the models against RHC-mPAP (Fig. 4), this was not performed for alpha index as it was not designed to directly assess mPAP. Bland-Altman analysis showed modest accuracy for CMR-RV (bias $-5.2 \%$, limits of agreement -51.2 to $40.8 \%$ ) and CMR-PA/RV models (bias $12.2 \%$ and limits of agreement -30.7 to $55.0 \%$ ). Table 2 shows the correlation of the CMR models against RHC mPAP. Figure 5 shows the ROC curves for the three CMR models. CMR-PA/RV and CMR-RV had the largest AUC values, 0.93 (95\% confidence interval $0.86-1.0)$ and 0.91 (95\% confidence interval 0.84 $0.97)$, respectively, and alpha index also showed good diagnostic accuracy with AUC 0.837 (95\% confidence interval 0.74-0.94). The sensitivity and specificity of the CMR-PA/ RV model was $92 \%$ and $80 \%$, CMR-RV model $90 \%$ and $79 \%$, and the alpha index $100 \%$ and $13 \%$, respectively (Table 3).

\section{Outcome}

During a mean follow up period of 1.5 years (standard deviation 0.9) there were 33 deaths. Table 4 gives the scaled univariate Cox proportional hazards regression results for patient demographics, RHC and CMR measurements. Age, sex, walk distance FEV1 percent predicted, FVC percent predicted and FEV1/FVC ratio were not univariate predictors of mortality. PA relative area change, right ventricular mass index, ventricular mass index, interventricular septal angle and RV enddiastolic volume index were univariate predictors of mortality ( $p=0.009, p<0.001, p=0.002$ and $p=0.023$, respectively). RHC measured mPAP was a strong predictor of mortality ( $p=0.004$ ), as were the CMR-RV and CMR-PA/RV models ( $p=0.002$ and $p=0.012$, respectively). There was a statistically significant difference in survival when the population was split by a threshold of $35 \mathrm{mmHg}$ for RHC-mPAP, CMR-RV and CMR-PA/RV models, with a log rank chi squared of 5.03, 5.47 and 7.10, respectively (see Fig. 6 and Table 5). On multivariate analysis (performed on all univariate predictors of 

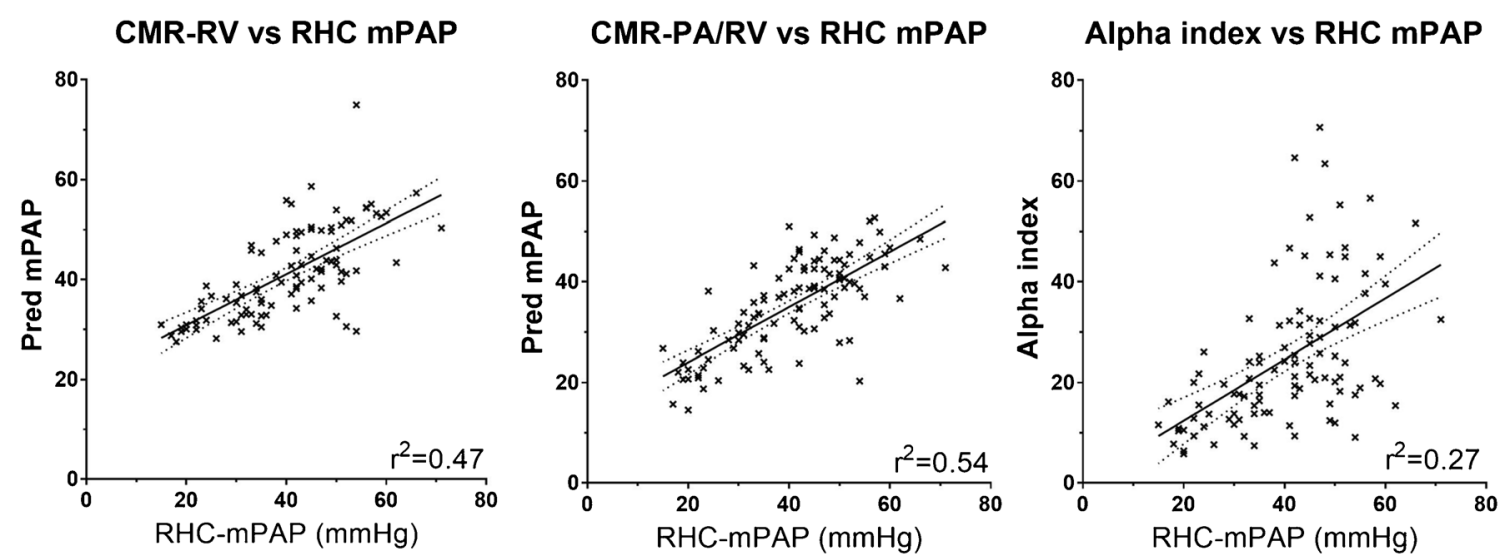

Fig. 3 Scatter plots showing correlations of each MRI model with right heart catheter measured mPAP

outcome for demographics, right heart catheter and cardiac MRI), right ventricular mass index and PA relative area change were statistically significant, with scaled Cox multivariate hazard ratios $1.549(95 \%$ CI 1.172-2.047, $p=0.002)$ and 0.561 (95\% CI $0.333-0.946, p=0.030)$, respectively.

\section{Discussion}

This study demonstrates that CMR models can be used to assess for the presence of pulmonary hypertension in COPD with good diagnostic accuracy, whilst also providing prognostic information similar to right heart catheterisation. This data supports the potential use of CMR in the non-invasive assessment of suspected pulmonary hypertension in patients with COPD. We have shown that single measurements from cardiac MRI (most notably septal angle) correlate strongly with right heart catheter measured mPAP. A model which includes measurements from the right ventricle, the diagnostic accuracy is slightly reduced, but inclusion of basic measurements of pulmonary arterial structure and function increases the accuracy of predicted mPAP and, therefore, the presence of pulmonary hypertension. Therefore, we recommend the use of CMR-PA/RV model in the diagnosis of pulmonary hypertension in patients with COPD. In the presence of COPD, right ventricular mass index and PA relative area change are the strongest predictors of outcome, but further work to develop prognostic thresholds is required.

Studies performed primarily in patients with pulmonary arterial hypertension have shown that structural and functional indices of the right ventricle (RV end diastolic volume index, $\mathrm{RV}$ end-systolic volume index, RV ejection fraction, RV mass, interventricular septal angle) and pulmonary artery (PA area, PA relative area change and black blood score) correlate with RHC measured mPAP. Inter- and intra-observer reproducibility data for these cardiac MRI metrics for DC and AJS have been previously published. RV end-diastolic and systolic measurements had excellent inter-observer reproducibility (ICC 0.959 and 0.991 ) and pulmonary artery relative area change had high inter-observer reproducibility (ICC 0.891). Right ventricular ejection fraction also had high inter-observer reproducibility (ICC 0.957) [22]. We and others, have shown that we can further develop predictive equations using MR metrics to estimate measurements made at cardiac
Fig. 4 Bland-Altman plots showing accuracy of models against RHC-mPAP
CMR-RV vs MPAP

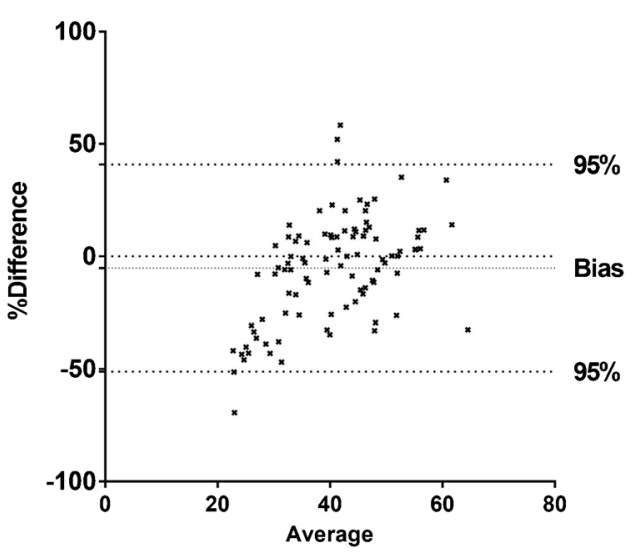

CMR-PA/RV vs MPAP

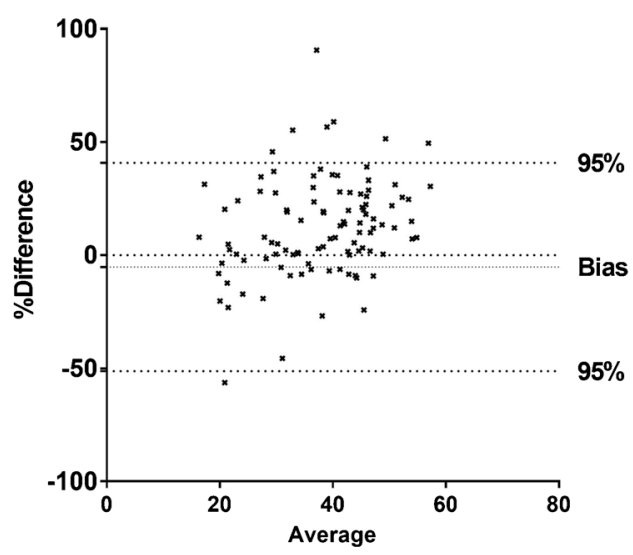




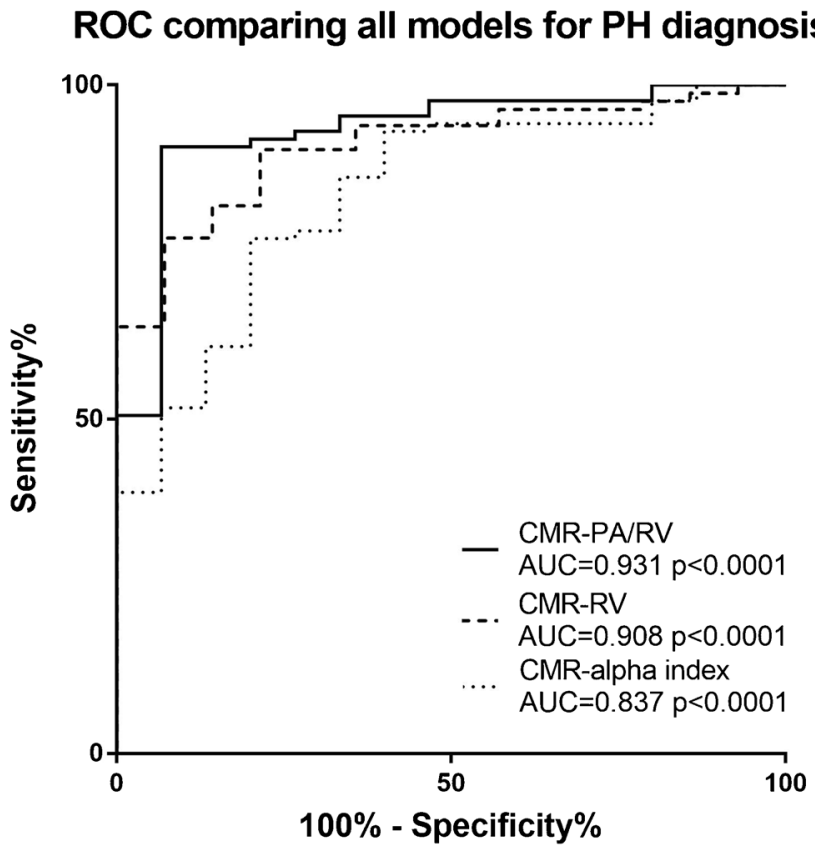

Fig. 5 ROC curve for the diagnosis of pulmonary hypertension

catheterisation. However, to date there is very little data on the use of MR imaging as a diagnostic and prognostic tool in patients with COPD and suspected pulmonary hypertension. In this study we have shown that these structural and functional indices of the right ventricle and pulmonary artery correlate with right heart catheter measured mean pulmonary arterial pressure: with pulmonary artery relative area change, ventricular mass index and interventricular septal angle having the strongest correlations. We have shown that the use of CMR-PA/RV and CMR-RV models, improve the prediction of mPAP and also the diagnostic accuracy of quantitative CMR measures.

The alpha model showed good correlation with mPAP, but had a low diagnostic accuracy: this model suffered from a diagnostic threshold that was too low for this cohort, significantly reducing its specificity. Changing the threshold of the alpha index to 16 increased the diagnostic accuracy of this model to $77 \%$ sensitivity and $80 \%$ specificity. This inaccuracy may be related to the measurement of right ventricular ejection fraction, particularly the reproducibility between different centres. In the original paper, it was not described how the right ventricular endocardial contours were measured, specifically, whether the papillary muscles and RV trabeculations were included, which may potentially add a bias into the calculation.

Pulmonary hypertension, confirmed by right heart catheter, has been recognised for many years as a marker of disease severity in COPD. In this study we have shown that cardiac MRI measured right ventricular mass and therefore ventricular mass index, along with pulmonary artery relative area change are significant predictors of mortality on univariate analysis. 
Table 4 Univariate Cox proportional hazards regression analysis for survival

\begin{tabular}{|c|c|c|c|c|}
\hline & Cox univariate hazard ratio & \multicolumn{2}{|c|}{$95 \%$ confidence interval } & $p$-value \\
\hline \multicolumn{5}{|l|}{ Demographics } \\
\hline Age & 1.424 & 0.936 & 2.166 & 0.098 \\
\hline Sex & 0.781 & 0.388 & 1.57 & 0.487 \\
\hline WHO functional status & 1.45 & 1.031 & 2.039 & 0.033 \\
\hline Walk distance & 0.475 & 0.167 & 1.354 & 0.164 \\
\hline FVC \% pred & 1.221 & 0.835 & 1.785 & 0.304 \\
\hline FEV1 \% pred & 1.399 & 0.928 & 2.108 & 0.109 \\
\hline FEV1/FVC & 1.244 & 0.85 & 1.82 & 0.262 \\
\hline TLCO \% pred & 0.593 & 0.19 & 1.852 & 0.369 \\
\hline \multicolumn{5}{|l|}{ Right heart catheter } \\
\hline mPAP & 1.74 & 1.198 & 2.526 & 0.004 \\
\hline mRAP & 1.297 & 0.873 & 1.927 & 0.199 \\
\hline PAWP & 0.933 & 0.651 & 1.338 & 0.707 \\
\hline $\mathrm{CI}$ & 0.847 & 0.566 & 1.268 & 0.421 \\
\hline PVRI & 1.384 & 1.034 & 1.853 & 0.029 \\
\hline $\mathrm{SvO} 2$ & 0.857 & 0.59 & 1.245 & 0.418 \\
\hline \multicolumn{5}{|l|}{ Cardiac MRI } \\
\hline RV EDV index & 1.381 & 1.013 & 1.882 & 0.041 \\
\hline RV ESV index & 1.338 & 0.981 & 1.824 & 0.066 \\
\hline RVEF & 0.784 & 0.548 & 1.122 & 0.183 \\
\hline RV mass index & 1.673 & 1.303 & 2.148 & $<0.001$ \\
\hline LV EDV index & 0.759 & 0.494 & 1.166 & 0.209 \\
\hline LV ESV index & 0.766 & 0.478 & 1.228 & 0.269 \\
\hline LVEF & 1.077 & 0.756 & 1.535 & 0.681 \\
\hline PA RAC & 0.543 & 0.343 & 0.859 & 0.009 \\
\hline VMI & 1.417 & 1.135 & 1.768 & 0.002 \\
\hline IVS angle & 1.48 & 1.055 & 2.075 & 0.023 \\
\hline Black blood score & 1.377 & 0.925 & 2.05 & 0.115 \\
\hline Average PA velocity & 1.018 & 0.697 & 1.484 & 0.928 \\
\hline Alpha-index & 1.19 & 0.858 & 1.65 & 0.297 \\
\hline CMR-RV & 1.565 & 1.175 & 2.083 & 0.002 \\
\hline CMR-PA/RV & 1.59 & 1.108 & 2.282 & 0.012 \\
\hline
\end{tabular}

WHO: World Health Organisation, FEV1: Forced expiratory volume in $1 \mathrm{~s}$, FVC: Forced vital capacity, RHC: right heart catheter, mPAP: right heart catheter measured mean pulmonary artery pressure, mRAP: mean right atrial pressure, PAWP: pulmonary artery wedge pressure, CI: cardiac index, PVRI: pulmonary vascular resistance index, $\mathrm{SvO}_{2}$ : mixed venous oxygen saturation, $\mathrm{CMR}$ : cardiopulmonary magnetic resonance, RVEDV: right ventricular end-diastolic volume, RVESV: right ventricular end-systolic volume, RVEF: right ventricular ejection fraction, RV: right ventricle, LVEDV: left ventricular end-diastolic volume, LVESV: left ventricular end-systolic volume, LVEF: left ventricular ejection fraction, PA RAC: pulmonary artery relative area change, VMI: ventricular mass index, IVS: interventricular septum, CMR-RV: right ventricle based cardiopulmonary magnetic resonance image model, CMR-PA/RV: pulmonary artery and right ventricle based cardiopulmonary magnetic resonance image model
However, by using CMR derived parametric models, particularly the CMR-RV model which combine these CMRI derived measures, we can improve the prediction of outcome in COPD patients on multivariate analysis. These models may be further improved with novel imaging biomarkers in pulmonary hypertension assessment. One such marker is the 4D flow assessment of the life of vortices within the pulmonary artery, which has been shown to have a very high correlation with mPAP ( $\mathrm{r}^{2}$ 0.95) [23].

A potential source of error arises from the use of MR models, which were developed for use in a cohort of all causes of pulmonary hypertension and not validated in specific subgroups, such as PH-COPD in this case. We feel that it is likely that the findings of a raised interventricular septal angle and 
Survival by RHC-mPAP

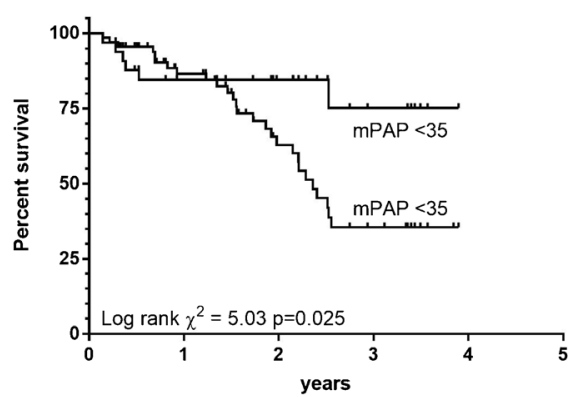

Survival by CMR-RV

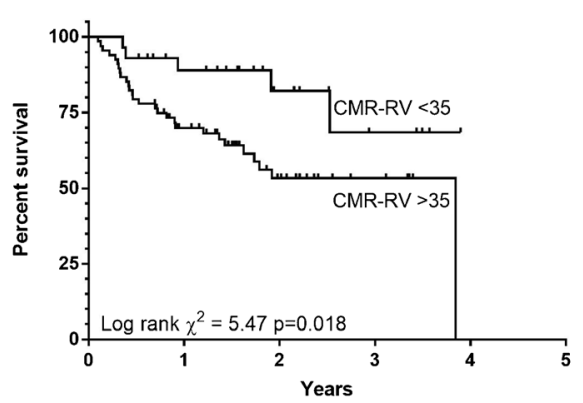

Survival by CMR-PARV

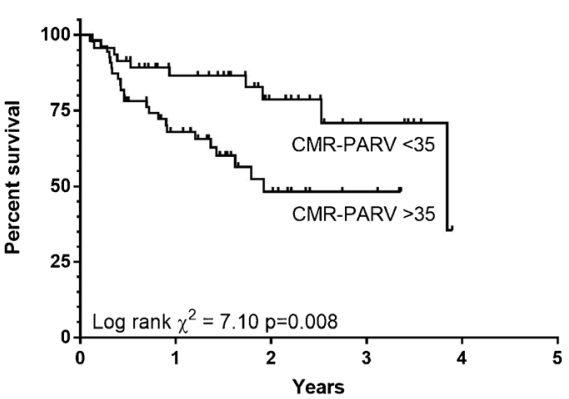

Fig. 6 Kaplan-Meier survival tables, all dichotomised by 35

ventricular mass index are likely to occur most subgroups of pulmonary hypertension (with the exception of left heart disease) as they are related to the pressure differential between the left and right ventricles. The measures of pulmonary arterial structure and function (size and relative area change) are likely to be transferable across subgroups, as they are markers of pulmonary vascular compliance and remodelling. We, therefore, feel that it reasonable to use these models in specific sub-groups of pulmonary hypertension, although validation, such as in this paper, would be useful. The models that are used in this paper use parameters that are stated with a degree of precision (for example an offset of 21.806 for CMR-PA/ $\mathrm{RV})$, likely more than is required for this purpose. We have maintained the equations in the published form to reduce any bias in the calculations, but it is likely that fewer decimal places could be used for these parameters for the prediction of outcome and the presence of pulmonary hypertension in COPD.

Currently, RHC is the gold standard test for the assessment of pulmonary hypertension and is used to assess prognosis in suspected COPD-PH cases. The ability to use non-invasive CMR models in this patient group may avoid the need for RHC, as we have shown CMR to be as good in the assessment of prognosis. The strong correlation of CMR imaging models with RHC mPAP suggest it may also have a role in follow-up, and also in assessment of treatment response in possible future trials assessing vasodilator response in COPD-PH. The role of cardiac MRI in COPD is further strengthened as this is a cohort of patients in which echocardiography is challenging. In the presence of COPD echocardiography has a relatively high rate of non-diagnostic quality studies. It has a good negative predictive value, but a poorer positive predictive value [14]. In patients with a normal right ventricle and predicted systolic pulmonary artery pressure (estimated from the tricuspid regurgitant jet velocity using the Bernoulli equation) pulmonary hypertension is highly unlikely. The main role for cardiac MRI in patients with COPD, therefore, probably lies in the group of patients with high estimated systolic pulmonary artery pressures or non-diagnostic scans. This may be at diagnosis, as studied in this paper, or potentially at follow-up.
The non-invasive estimation of mPAP has multiple other potential uses, beyond prognosis in COPD, although these have not been addressed in this paper. In advanced COPD patients considered for lung volume reduction surgery (LVRS), severe pulmonary hypertension is considered a contraindication [24], so the non-invasive assessment of mPAP on MR would be a useful tool in patient selection. Furthermore, left or right ventricular dysfunction is considered a relative contraindication to LVRS [24], and can be assessed at the same CMR sitting. In COPD patients with pulmonary hypertension there is evidence that outcome may be improved if treated with lung transplantation over LVRS [25], assessment of mPAP could be performed in the same sitting as a ventilation study in the preoperative assessment of LVRS or transplant candidates [26, 27], allowing for the assessment of the best surgical option and the best target in the lung.

This study is limited by its single centre, retrospective design, at a tertiary pulmonary hypertension referral centre where the severity of pulmonary hypertension is more severe. The results, therefore, represent a biased population, as the patients have been screened by echocardiography and excessive symptoms for the presence of pulmonary hypertension before referral (the vast majority of patients with COPD are managed in primary care). This has resulted in a smaller proportion of non-pulmonary hypertension patients than would be present in a general population of COPD; however, in the absence of a prospective trial, this is difficult to overcome. Furthermore, the assessment of the presence of COPD in the population relied upon the accurate documentation of a clinical diagnosis of COPD. This likely resulted in a lower proportion of patients being identified, but is likely to be more robust than using either spirometry or CT defined emphysema alone. Despite this, we feel that these tools are valid for the assessment of patients who are referred to a pulmonary hypertension centre regarding the presence of pulmonary hypertension and prognosis, as this is the population that was studied. It may be that more specific diagnostic thresholds would be suitable for use in the general COPD population. 


\section{Conclusion}

The use of the CMR-PA/RV model, derived from cardiovascular magnetic resonance imaging in the assessment of COPD allows for accurate, non-invasive estimation of pulmonary artery pressure and the presence of pulmonary hypertension. Right ventricular mass index and pulmonary artery relative area change are most predictive of outcome.

\section{Compliance with ethical standards}

Guarantor The scientific guarantor of this publication is Dr Andrew Swift.

Conflict of interest The authors of this manuscript declare no relationships with any companies, whose products or services may be related to the subject matter of the article.

Funding This work was supported by NIHR grant NIHR-RP-R3-12027, Wellcome grant 205188/Z/16/Z and MRC grant MR/M008894/1. The views expressed in this publication are those of the author(s) and not necessarily those of the NHS, the National Institute for Health Research or the Department of Health. D. Capener was part funded by an unrestricted research grant from Bayer.

Statistics and biometry No complex statistical methods were necessary for this paper.

Informed consent Written informed consent was waived by the Institutional Review Board.

Ethical approval Institutional Review Board approval was obtained.

\author{
Methodology \\ - retrospective \\ - diagnostic or prognostic study \\ - performed at one institution
}

Open Access This article is distributed under the terms of the Creative Commons Attribution 4.0 International License (http:// creativecommons.org/licenses/by/4.0/), which permits unrestricted use, distribution, and reproduction in any medium, provided you give appropriate credit to the original author(s) and the source, provide a link to the Creative Commons license, and indicate if changes were made.

\section{References}

1. Chaouat A, Naeije R, Weitzenblum E (2008) Pulmonary hypertension in COPD. Eur Respir J 32:1371-1385.

2. Wright JL (2005) Pulmonary hypertension in chronic obstructive pulmonary disease: current theories of pathogenesis and their implications for treatment. Thorax 60:605-609.

3. Andersen KH, Iversen M, Kjaergaard J et al (2012) Prevalence, predictors, and survival in pulmonary hypertension related to endstage chronic obstructive pulmonary disease. J Hear Lung Transplant 31:373-380.

4. Burrows B, Kettel LJ, Niden AH et al (1972) Patterns of Cardiovascular Dysfunction in Chronic Obstructive Lung Disease. N Engl J Med 286:912-918.
5. Cooper R, Ghali J, Simmons BE, Castaner A (1991) Elevated Pulmonary Artery Pressure. Chest 99:112-120.

6. Oswald-Mammosser M, Weitzenblum E, Quoix E et al (1995) Prognostic Factors in COPD Patients Receiving Long-term Oxygen Therapy: Importance of Pulmonary Artery Pressure. Chest 107:1193-1198.

7. Minai OA, Chaouat A, Adnot S (2010) Pulmonary Hypertension in COPD: Epidemiology, Significance, and Management. Chest 137: 39S-51S.

8. Hyduk A, Croft JB, Ayala C et al (2005) Pulmonary hypertension surveillance-United States, 1980-2002. MMWR Surveill Summ 54:1-28

9. Seeger W, Adir Y, Barberà JA et al (2013) Pulmonary Hypertension in Chronic Lung Diseases. J Am Coll Cardiol 62:D109-D116.

10. Boerrigter BG, Bogaard HJ, Trip P et al (2012) Ventilatory and Cardiocirculatory Exercise Profiles in COPD. Chest 142:11661174.

11. Hurdman J, Condliffe R, Elliot CA et al (2013) Pulmonary hypertension in COPD: results from the ASPIRE registry. Eur Respir $\mathrm{J}$ 41:1292-1301.

12. Hoeper MM, Lee SH, Voswinckel R et al (2006) Complications of Right Heart Catheterization Procedures in Patients With Pulmonary Hypertension in Experienced Centers. J Am Coll Cardiol 48:25462552.

13. Fisher MR, Criner GJ, Fishman AP et al (2007) Estimating pulmonary artery pressures by echocardiography in patients with emphysema. Eur Respir J 30:914-921.

14. Arcasoy SM, Christie JD, Ferrari VA et al (2003) Echocardiographic Assessment of Pulmonary Hypertension in Patients with Advanced Lung Disease. Am J Respir Crit Care Med 167:735-740.

15. Swift AJ, Rajaram S, Hurdman J et al (2013) Noninvasive Estimation of PA Pressure, Flow, and Resistance With CMR Imaging. JACC Cardiovasc Imaging 6:1036-1047.

16. Swift A, Lungu A, Walker H, Capener D, Hammerton C, Elliot C, Condliffe R, Kiely D, Wild J (2015) Improved diagnostic accuracy of MRI in patients with suspected pulmonary hypertension with combined right ventricle and pulmonary artery metrics. ERS Int Congr 2015

17. Moral S, Fernández-Friera L, Stevens G et al (2012) New index alpha improves detection of pulmonary hypertension in comparison with other cardiac magnetic resonance indices. Int J Cardiol 161: 25-30.

18. Hurdman J, Condliffe R, Elliot CA et al (2012) ASPIRE registry: Assessing the Spectrum of Pulmonary hypertension Identified at a REferral centre. Eur Respir J 39:945-955.

19. Hansell DM, Bankier AA, MacMahon H et al (2008) Fleischner Society: Glossary of Terms for Thoracic Imaging. Radiology 246: 697-722.

20. Saba TS, Foster J, Cockburn M et al (2002) Ventricular mass index using magnetic resonance imaging accurately estimates pulmonary artery pressure. Eur Respir J 20:1519-1524.

21. Swift AJ, Rajaram S, Condliffe R et al (2012) Pulmonary artery relative area change detects mild elevations in pulmonary vascular resistance and predicts adverse outcome in pulmonary hypertension. Invest Radiol 47:571-577.

22. Swift AJ, Capener D, Johns CS et al (2017) Magnetic Resonance Imaging in the Prognostic Evaluation of Patients with Pulmonary Arterial Hypertension. Am J Respir Crit Care Med. https://doi.org/ 10.1164/rccm.201611-2365OC

23. Reiter G, Reiter U, Kovacs G et al (2015) Blood flow vortices along the main pulmonary artery measured with MR imaging for diagnosis of pulmonary hypertension. Radiology 275:71-79.

24. DeCamp MM, Lipson D, Krasna M et al (2008) The Evaluation and Preparation of the Patient for Lung Volume Reduction Surgery. Proc Am Thorac Soc 5:427-431. 
25. Meyers BF (2003) Chronic obstructive pulmonary disease * 10: Bullectomy, lung volume reduction surgery, and transplantation for patients with chronic obstructive pulmonary disease. Thorax 58:634-638.

26. Bauman G, Puderbach M, Deimling M et al (2009) Non-contrastenhanced perfusion and ventilation assessment of the human lung by means of Fourier decomposition in proton MRI. Magn Reson Med 62:656-664.

27. Swift AJ, Wild JM, Fichele S et al (2005) Emphysematous changes and normal variation in smokers and COPD patients using diffusion 3He MRI. Eur J Radiol 54:352-358. 